\title{
Changes of Bacterial Spectrum of Lower Respiratory Tract in Patients with Rhinovirus Induced AECOPD
}

\author{
Zhoufang Mei ${ }^{1}$, Tiping Yin ${ }^{2}$, Jiawei $\mathrm{Gu}^{3}$, Tianyun Shi ${ }^{1}$, Yong $\mathrm{Du}^{1}$, Jindong Shi ${ }^{1}$, Zhijun Jie ${ }^{1}$ \\ ${ }^{1}$ Department of Pulmonary and Critical Care Medicine, The Fifth People's Hospital of Shanghai, Fudan University, \\ Shanghai, 200240, China; ${ }^{2}$ Department of Pulmonary and Critical Care Medicine, Maanshan Central Hospital, \\ Maanshan, 243000, China; ${ }^{3}$ Department of Clinical Medicine, Shanghai Medical School, Fudan University, \\ Shanghai, 200240, China
}

Zhoufang Mei and Tiping Yin are co-first authors. Zhoufang Mei MD, Department of Pulmonary and Critical Care Medicine, The Fifth People's Hospital of Shanghai, Fudan University, Shanghai, 200240, China. E-mail: meizhoufang@163.com.

Corresponding Author: Zhijun Jie, Department of Pulmonary and Critical Care Medicine, The Fifth People's Hospital of Shanghai, Fudan University, Shanghai, 200240, China. mail:jiezjlxh @ 163.com

The study was funded by the Shanghai Health and Family Planning Commission (No. 20174Y0048).

Abbreviation: AECOPD, acute exacerbation of chronic obstructive pulmonary disease; RhV, rhinovirus.

Received February 28, 2019; Accepted March 17, 2019

\begin{abstract}
Objective: To evaluate the bacteria pathogenic spectrum in the lower respiratory tract of Acute Exacerbated Chronic obstructive pulmonary diseases (AECOPD) patient with Rhinovirus infection. Methods: Sputum samples were collected from patients with AECOPD hospitalized. Respiratory viruses were detected by fluorescent quantitative PCR. According to the results of RhV detection, the patients were divided into two groups: AECOPD patients with rhV positive (RhV+AECOPD group) and AECOPD patients with rhV negative (RhV-AECOPD group) with 11 cases in each group. DNA was extracted from patient sputum. Meta rDNA amplification library was constructed and validated. Variable V4 region of 16S rDNA gene was sequenced. Operational taxonomic unit (OTU) and bacterial species were analyzed between the two groups. Results: There was no significant difference in the average OTUs between the two groups. According to the classification of bacterial pathogens, the level of actinomycetes in sputum of RhV +AECOPD group was significantly higher than that of RhV-AECOPD group $(P<0.05)$; Corynebacterium, Neisseria and Roxiella had significant difference in genus level between the two groups; The species level of Roxiella mucosa had a significant difference in the two groups. The differences between the two groups, from the specific results of complements, indicated that the bacterial pathogens in the experimental group were less than those in the control group: The variable $V 4$ region of $16 \mathrm{~S}$ rDNA gene sequenced by two-index and two-terminal sequencing, showed that there was the difference in the diversity of lower respiratory tract bacteria between the two groups, indicating that there were significant differences in the microbial community structure between the two groups.
\end{abstract}

Key Words: Rhinovirus; chronic obstructive pulmonary disease; exacerbation; microorganism.

COPD is a chronic inflammatory disease within the respiratory tract. The incidence of COPD is increasingly common in recent years. It is expected that COPD will become the top four contributors to mortality in the world by $2020 .{ }^{1}$

AECOPD can further aggravate the pulmonary function problem, leading to the decline of patients life quality-life and the increase in the mortality rate. ${ }^{2}$ Most of the AE COPD patients relate to infection, and $40 \%$ of the AECOPD relate to RhV-based viral infection. ${ }^{3}$ In addition, co-infection of viruses and bacteria can be detected in $25 \%$ of acute exacerbated COPD cases, and studies have shownthat co-infection of viruses and bacteria canfurther aggravate the lung function problem and prolong hospitalization time. ${ }^{4,5}$
This is an open access journal, and articles are distributed under the terms of the Creative Commons Attribution-NonCommercial-ShareAlike 4.0 License, which allows others to remix, tweak, and build upon the work non-commercially, as long as appropriate credit is given and the new creations are licensed under the identical terms.

For reprints contact: weda-h@weda-h.org

How to cite this article: Mei ZF, Yin TP, Gu JW, Shi TY, Du Y, Shi JD, Jie ZJ. Changes of Bacterial Spectrum of Lower Respiratory Tract in Patients with Rhinovirus Induced AECOPD. J ADV HEALTH 2019; 1(2): 96-103. 
Respiratory microorganisms themselves are believed to be beneficial for the host to promote the differentiation of mucosal structure and function, stimulate innate immunity and adaptive immune response, and resist the invasion of pathogens through the "anti-colonization" effect. ${ }^{6}$ Virus infection may break the immune balance between host and respiratory microorganisms, it creates favorable conditions for the growth of pathogenic bacteria. About $60 \%$ of COPD patients had secondary sputum bacterial culture positive after RhV infection. ${ }^{7}$

Molyneaux et al. ${ }^{3}$ Longitudinally compared the changes of respiratory flora before and after $\mathrm{RhV}$ infection in COPD patients, in order to elaborate on the influence of $\mathrm{RhV}$ on respiratory flora. This study compared the bacterial spectrum of the lower respiratory tract between RhV-positive AECOPD patients and virus-negative AECOPD patients, it reveals the differences of bacterial flora between two testing groups, and provide guidance for clinical prevention and treatment of secondary bacterial infections.

\section{Materials and methods}

\section{Ethical description}

The study was approved by the Ethics Committee of Shanghai Fifth People's Hospital. All the volunteers have signed written consent.

\section{Study subjects}

Patients admitted from the Fifth People's Hospital of Shanghai in June 2015 -2016 in May. Sputum samples were collected from patients without treatment on the first day of admission. The sputum of all patients was screened and detected by fluorescent quantitative PCR in Shanghai Public Health Clinical Center for 10 respiratory viruses, including influenza virus type A (FluA), influenza virus type $\mathrm{B}$ (FluB), adenovirus (ADV), respiratory syncytial virus type A (RSV-A). (respiratory syncytial virus type A, RSVA), respiratory syncytial virus type B (RSVB), rhinovirus $(\mathrm{RhV})$, parainfluenza virus (PIV), coronavirus (CoV), Boca virus (BoV), and metapneumonia virus (m). Etapneumovirus, MPV). Eleven $\mathrm{RhV}$ positive sputum samples from patients with AECOPD were selected as the experimental group and 11 virus negative sputum samples as control group.

\section{Entry criteria and exclusion criteria}

Inclusion criteria: all patients met the diagnostic criteria ${ }^{8}$ for COPD guidelines. Acute exacerbation of COPD is defined as the continuous deterioration of COPD patients beyond their daily conditions and the need to change the routine medication of basic COPD. In the course of the disease, cough, sputum, shortness of breath and/or wheezing are aggravated in the short term, sputum volume is increased, purulent or viscous purulent, and inflammation such as fever can be significantly aggravated.

Exclusion criteria: Patients who cannot cooperate with pulmonary function tests, those who do not meet the definition of acute exacerbation of COPD mentioned above, those with asthma, bronchiectasis, cystic fibrosis, active pulmonary tuberculosis or other respiratory diseases requiring treatment or diagnosis, those with mental disorders or any related immune inflammation reaction. Other diseases and patients who received antibiotics or hormone therapy during the past 4 weeks. In the control group, 1 sample was removed after it was detected that it did not meet the requirement of building meta rDNA amplification library.

\section{Collection of clinical data}

The general information of patients, including age, sex, smoking history and complications, laboratory and examination results, including blood routine, liver function, coagulation items, blood gas analysis, antibiotic use time, prognosis and hospitalization time were collected.

\section{Experimental method}

\section{(1) methods for sample testing}

The sputum metagenic DNA was extracted according to the standard operating procedure of Meta DNA extraction, and the sample concentration was quantified accurately by the qubit fluorimetry. The sample integrity was detected by agarose gel electrophoresis (agarose concentration: 1\%; voltage: $150 \mathrm{~V}$; electrophoresis time: $40 \mathrm{~min}$ ).

\section{(2) building Meta rDNA amplicon Library}

Genomic DNA was standardized to $30 \mathrm{ng}$ for each PCR reaction, and then the primer mixture of V4 double index fusion PCR and the precursor of PCR (NEB Phusion high fidelity PCR) were added to run the PCR. The fusion temperature is $56 \mathrm{C}$ and the PCR cycle is 30 times. PCR products remove non-specific products with Ampure XP beads (AGINCOURT).

(3) verify the Meta rDNA amplicon library.

The final library was quantified in two ways: using the Agilent 2100 Bioanalyzer (Agilent). The average molecular length was determined by DNA 1000 reagent and the library was quantified by real-time quantitative PCR (Eva GreenTM).

(4) sequencing of Meta rDNA amplicon Library

Qualified libraries will be sequenced in the MiSeq system using the sequencing strategy PE250 (PE251+8+8+251) (MiSeq kit) by double exponential double-ended sequencing of the variable V4 region of $16 \mathrm{~S}$ rDNA gene.

(5) bioinformatics analysis

During data processing and filtering, we used the internal written procedures of the Chinese big gene company through Illumina

The platform (Hiseq) carries out Paired-end sequencing to filter low-quality reads and obtain high-quality Clean Data. Sequence splicing software FLASH v1.2.11 is used to assemble pairs of reads obtained by double-terminal sequencing into a sequence and obtain Tags with the high variable region. Tags were clustered into OTUs by USEARCH (v7.0.1090). Then the OTU representative sequences were compared with the database Release 9201203 by RDP classifier (v2.2). Species complexity and species diversity were analyzed based on OTU and species annotation results.

(6) statistical analysis

Discrete variables are represented by counting (percentage) and continuous variables by means of mean + standard deviation. The $t$ test is used to compare the mean of two independent samples, and Fisher's exact probability method is used to compare discrete variables. Alpha diversity analysis is the analysis of species diversity in a single sample. ACE index, Chao index, Shannon index and Simpson index are used to evaluate species diversity. Beta diversity analysis is used to compare the differences in species diversity between a pair of samples, and Bray-Curtis distance is used to assess the differences. Alpha diversity and Bray-Curtis distance are calculated by Mothur (v.1.27). $\mathrm{P}<0.05$ is considered to be 
meaningful in all statistical analyses. SPSS 18 and GraphPad. cameyo 6 are used for data analysis and processing.

\section{Result}

\section{Clinical characteristics analysis}

The clinical characteristics of AECOPD patients in the rhinovirus positive group (11 cases) and the virus negative group (10 cases) were compared, the characters include age, sex, smoking history, complications, body temperature, blood routine, liver function, coagulation function, antibiotic usage time, prognosis and hospitalization time. The result shows that there are no significant statistical differences to be found. The differences was $(\mathrm{P}>0.05)$ (see Table 1).

\section{OTUs diversity analysis}

We generated an average of 25,587 sequences from RhV-positive AECOPD patients and 27,654 sequences from virus-negative AECOPD patients, and the corresponding OTUs averaged 132 and 134 in $97 \%$ similarity between the two groups, but there was no statistical difference between the two groups (see Figure 1). The alpha diversity analysis (such as ACE index, Chao index, Shannon index and Simpson index) was presented in Table 2. The comparison of alpha diversity index between the two groups showed no statistical difference. Beta diversity was analyzed by Bray-Curtis clustering tree graph (see Figure 2). In this graph, no $\mathrm{RhV}$ positive or virus negative samples were found clustering in a branch of the tree. Two groups of samples were intersected and distributed in different branches.

\section{Microbial composition.}

In this study, the total sequence measured provided a taxonomic map of sputum microorganisms showing 20 phyla, 41 classes, 65 orders, 108 families, 160 genera and 62 species.

At the door level, 20 doors were presented in sputum samples (see Fig. 3a). Higher taxa included the posterior wall bacteria (42.34\% of RhV-positive microorganisms and $45.91 \%$ of virus-negative microorganisms), Bacteroides $(27.35 \%$ and $22.71 \%$, respectively), Proteus $(20.23 \%$ and $21.66 \%$, respectively), Fusobacterium (4.60\% and $4.07 \%$, respectively) and Actinomycetes (2.67\% and $1.55 \%$, respectively). The gates in the two groups constitute $97.19 \%$ and $95.90 \%$ of the total sequence respectively. Only RhV positive group was found to be verruca microphylla and GNO2 gate, accounting for about $0.002 \%$ and $0.012 \%$ of total microorganism respectively. However, only in the viral negative group were found to be Curvularia and Phytophthora, accounting for about $0.002 \%$ and $0.004 \%$ of the microorganisms, respectively.

Table 1. comparison of clinical characteristics between rhinovirus positive and virus negative AECOPD patients

\begin{tabular}{|c|c|c|c|}
\hline Parameter & $\mathrm{RhV}$ positive AECOPD patients $(\mathrm{n}=11)$ & virus negative AECOPD patients $(\mathrm{n}=10)$ & Chi square value and $\mathrm{P}$ value \\
\hline Age (year) & $71 \pm 3.1$ & $70 \pm 2.8$ & $P=0.889$ \\
\hline Sex (male) & $8(72.73)$ & $7(70.00)$ & $P=1.000$ \\
\hline Smoking & $7(63.64)$ & $5(50.00)$ & $P=0.670$ \\
\hline Complications & $6(54.55)$ & $9(90.00)$ & $P=0.149$ \\
\hline Body temperature $\leqslant 37.3 \mathrm{C}$ & $10(90.91)$ & $8(80.00)$ & $P=0.587$ \\
\hline Body temperature $>37.3 \mathrm{C}$ & $1(9.09)$ & $2(20.00)$ & \\
\hline White blood cell count $\left(>10 \times 10^{\wedge} 9 / \mathrm{L}\right)$ & $2(18.18)$ & $1(10.00)$ & $P=1.000$ \\
\hline White blood cell count $\left(\leqslant 10 \times 10^{\wedge} 9 / \mathrm{L}\right)$ & $9(81.82)$ & $9(90.00)$ & \\
\hline Neutrophil count $\quad\left(10^{\wedge} 9 / \mathrm{L}\right)$ & $5.60 \pm 0.86$ & $4.91 \pm 0.55$ & $P=0.130$ \\
\hline Lymphocyte count $\quad\left(10^{\wedge} 9 / \mathrm{L}\right)$ & $1.62 \pm 0.32$ & $1.70 \pm 0.19$ & $P=0.839$ \\
\hline Eosinophils $\left(10^{\wedge} 9 / \mathrm{L}\right)$ & $0.14 \pm 0.09$ & $0.16 \pm 0.06$ & $P=0.887$ \\
\hline Hemoglobin $(\mathrm{g} / \mathrm{L})$ & $127 \pm 4.6$ & $120 \pm 5.7$ & $P=0.349$ \\
\hline $\operatorname{ESR}(\mathrm{mm} / \mathrm{h})$ & $33 \pm 8.0$ & $36 \pm 13.0$ & $P=0.710$ \\
\hline CRP (mg/L) & $17.06 \pm 7.15$ & $18.13 \pm 5.02$ & $P=0.906$ \\
\hline Procalcitonin (ng/mL) & $0.05 \pm 0.02$ & $0.07 \pm 0.03$ & $P=0.649$ \\
\hline D- two dimer（ug/L） & $0.67 \pm 0.16$ & $0.96 \pm 0.25$ & $P=0.197$ \\
\hline $\operatorname{ALT}(\mathrm{U} / \mathrm{L})$ & $20 \pm 4.9$ & $23 \pm 6.9$ & $P=0.447$ \\
\hline LDH (U/L) & $281 \pm 60.1$ & $271 \pm 40.1$ & $P=0.889$ \\
\hline $\mathrm{PH}$ & $7.42 \pm 0.01$ & $7.39 \pm 0.01$ & $P=0.169$ \\
\hline $\mathrm{PaO} 2(\mathrm{mmHg})$ & $89.6 \pm 6.7$ & $92.4 \pm 8.8$ & $P=0.802$ \\
\hline $\mathrm{PaCO} 2(\mathrm{mmHg})$ & $45.3 \pm 4.1$ & $48.4 \pm 2.5$ & $P=0.552$ \\
\hline $\mathrm{SAO} 2(\%)$ & $96.0 \pm 0.7$ & $96.2 \pm 0.8$ & $P=0.894$ \\
\hline PHT（mmHg） & $37 \pm 6.5$ & $45 \pm 7.5$ & $P=0.306$ \\
\hline HOD（d） & $10 \pm 0.9$ & $10 \pm 1.1$ & $P=0.844$ \\
\hline Days of antibiotic use (d) & $12 \pm 3.2$ & $11 \pm 3.6$ & $P=0.532$ \\
\hline Mortality (individual) & $0(0.00)$ & $0(0.00)$ & $P=1.000$ \\
\hline
\end{tabular}

Note: $P<0.05$ indicates that there is a significant difference between the two groups, with statistical significance; the above values are expressed by mean \pm standard deviation or counting (percentage), using t-test and Fisher's exact probability method. 
Table 2. Alpha Diversity Analysis of All Samples at 97\% Similarity

\begin{tabular}{|c|c|c|c|c|}
\hline Sample number & chao & ace & shannon & simpson \\
\hline RA1 & 134.5 & 136.20961 & 2.431979 & 0.136777 \\
\hline RA2 & 236.857143 & 240.663379 & 3.513141 & 0.057304 \\
\hline RA3 & 124.5 & 125.392 & 3.10924 & 0.098695 \\
\hline RA4 & 135.866667 & 132.962317 & 2.549182 & 0.122363 \\
\hline RA5 & 163.857143 & 155.244874 & 3.129914 & 0.099724 \\
\hline RA6 & 161.25 & 158.678073 & 2.347577 & 0.202257 \\
\hline RA7 & 166 & 166.163767 & 3.45852 & 0.064706 \\
\hline RA8 & 83 & 83.373446 & 2.365371 & 0.164682 \\
\hline RA9 & 195.2 & 169.37627 & 1.94734 & 0.28224 \\
\hline RA10 & 143.071429 & 143.739587 & 1.90196 & 0.415508 \\
\hline RA11 & 136.153846 & 133.412765 & 2.07753 & 0.239247 \\
\hline Al & 103 & 115.249581 & 1.930024 & 0.318995 \\
\hline $\mathrm{A} 2$ & 144.315789 & 157.026115 & 2.426431 & 0.145361 \\
\hline $\mathrm{A} 3$ & 121.909091 & 150.177887 & 1.22708 & 0.560757 \\
\hline $\mathrm{A} 4$ & 144.857143 & 145.370895 & 2.935694 & 0.115315 \\
\hline A5 & 59.111111 & 63.879509 & 0.832303 & 0.676633 \\
\hline A6 & 187.5 & 188.156065 & 3.493712 & 0.055526 \\
\hline A7 & 226.882353 & 221.113872 & 2.634352 & 0.257711 \\
\hline A8 & 180.071429 & 200.469099 & 3.114787 & 0.083656 \\
\hline A9 & 159.111111 & 158.687289 & 3.033778 & 0.148011 \\
\hline A10 & 201.076923 & 205.599802 & 2.570417 & 0.168522 \\
\hline
\end{tabular}

Note: RA1-RA11 was rhinovirus positive AECOPD group, A1-A10 was virus negative AECOPD group.

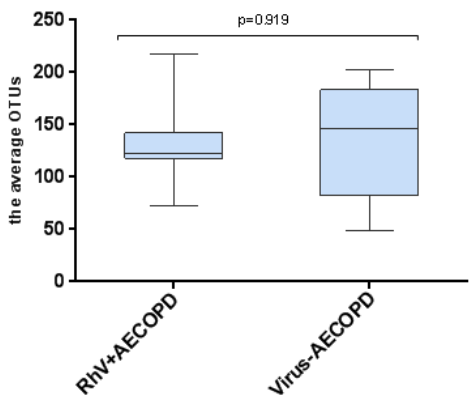

Figure 1. Comparison of average OTUs between rhinovirus-positive AECOPD and virus-negative AECOPD

Note: $\mathrm{P}<0.05$ indicates that there is statistical significance.

Abbreviations: AECOPD, acute exacerbation of chronic obstructive pulmonary disease; OTU, operational taxon; RhV, rhinovirus.

At the generic level, 160 genera were found in sputum samples, 127 genera were found in RhV positive group samples, and 145 genera were found in virus-negative group samples (see Figure 3b). Of these genera, 112 genera were shared among the two groups. Seven genera (Streptococcus, Puccinia, Neisseria, Chitinophaga, Oligotrophomonas, Veronococcus and Ciliarium) comprised about $69.20 \%$ of $\mathrm{RhV}$ positive samples and $52.67 \%$ of sputum microorganisms in virus-negative samples. A total of 20 genera (including the seven genera mentioned above and Streptococcus granulosus, Staphylococcus, Haemophilus, Roxiella, Clostridium, Staphylococcus, Carbonophilus, Lautropia, Sediminibacterium,

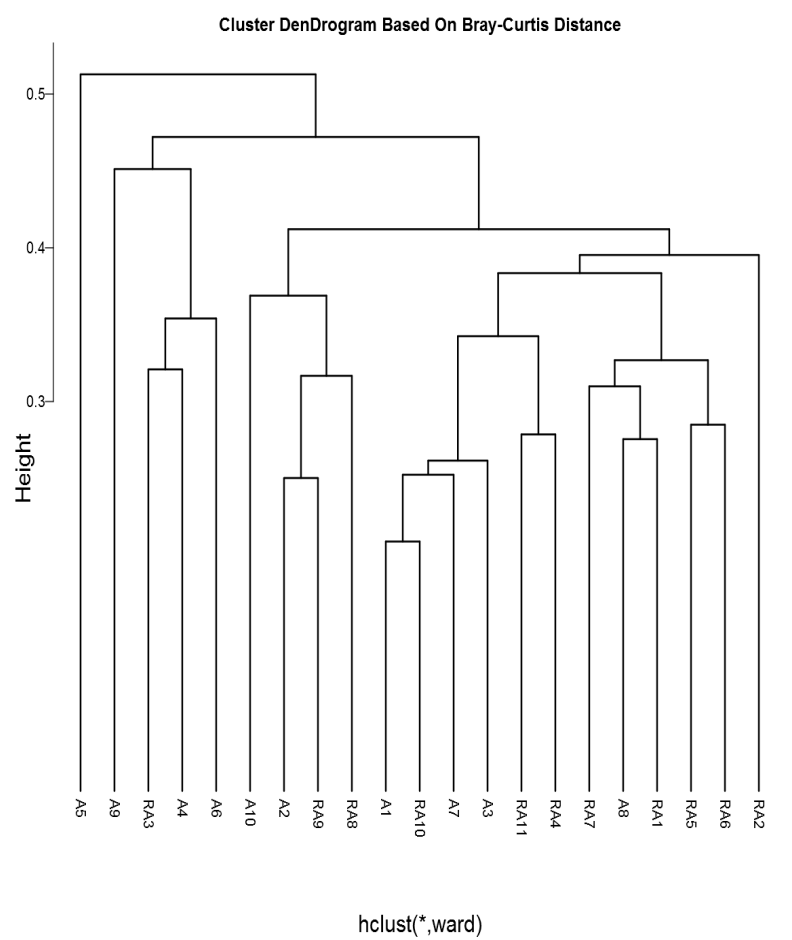

Figure 2. Bray-Curtis Cluster Tree of Subjects Note: RA1-RA11 was rhinovirus positive AECOPD group, A1-A10 was virus negative AECOPD group. 

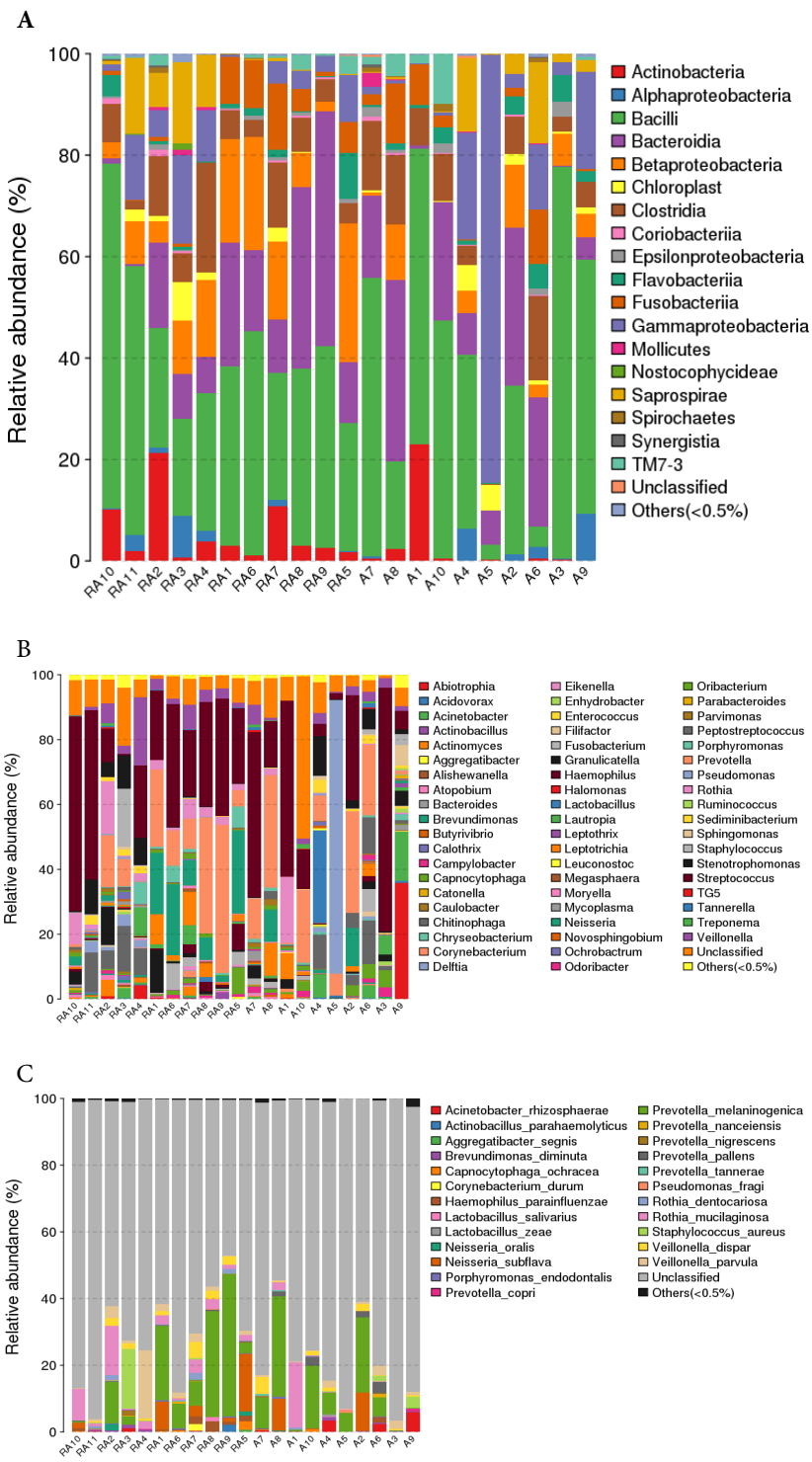

Figure 3. Abundance and prevalence of different phyla (A), genus (B) and species (C) in 21 samples

Note: RA1-RA11 was rhinovirus positive AECOPD group, A1-A10 was virus negative AECOPD group.

Oribacterium, Acinetobacter, Delft, Campylobacter) constitute $\mathrm{RhV}$ positive. There were about $87.51 \%$ microorganisms in the group and $66.36 \%$ in the virus negative group. Fifteen genera were detected only in $\mathrm{RhV}$ positive group, accounting for a relatively low abundance of microorganisms (about $0.102 \%$ ) and 33 genera were detected only in virus-negative group, accounting for a relatively low abundance of microorganisms (about $0.272 \%$ ).

At the species level, a total of 62 species were present, 50 species were found in $\mathrm{RhV}$ positive group samples and 58 species were found in virus-negative group samples (see Figure 3c). In these species, 46 species are shared in the two groups. Four genera were detected only in RhV positive group, which were ornithine-eating bacteriophage, anaerobic bacillus, Corynebacterium and Flavobacterium, accounting for the relatively low abundance of microorganisms (about $0.130 \%$ ). Twelve species were detected only in virus-negative group, which were arthrobacterium, bacteroides, Butyricicoccus, desulfurizing bacterium and purple bacillus.
Fragrant bacteria, psychrophilic bacteria, radish bacteria, rumen coccus-bromii, rumen coccus-flarefaciens, rumen coccus-gnavus and Streptococcus accounted for the relatively low abundance of microorganisms (about $0.151 \%$ ).

\section{Bacterial taxonomic analysis and comparison.}

Samples of microbes from rhinovirus positive group and virus negative group were also compared. Among all taxa, analysis at different taxonomic levels showed that the microorganisms in positive rhinovirus group were significantly higher than those in negative rhinovirus group $(P<0.05)$ : actinomycetes at the phylum level, actinomycetes at class level, actinomycetes and Neisseria at the order level, Corynebacteriaceae and Micrococcus at discipline level. Neisseriae and Corynebacteriaceae at the generic level, Neisseria and Rochella at the species level, Rochella mucinosa at the species level; microorganisms in the rhinovirus-positive group were significantly lower than those in the virus-negative group $(P<0.05)$ : Bdellovibrio at order level, Vibrio bacteriophage and Fibrobiophage at family level, and at generic level. It belongs to non-myxotropic fungi, bacteria, radish fungi and bacillus, and at the species level it belongs to radish fungi (see Fig. 4).

\section{Discussion}

Timely and accurate detection of bacterial spectrum within the lower respiratory tract is for understanding the interaction between viruses and bacteria in AECOPD and to prevent and treat secondary bacterial infections after virus infection. Early research
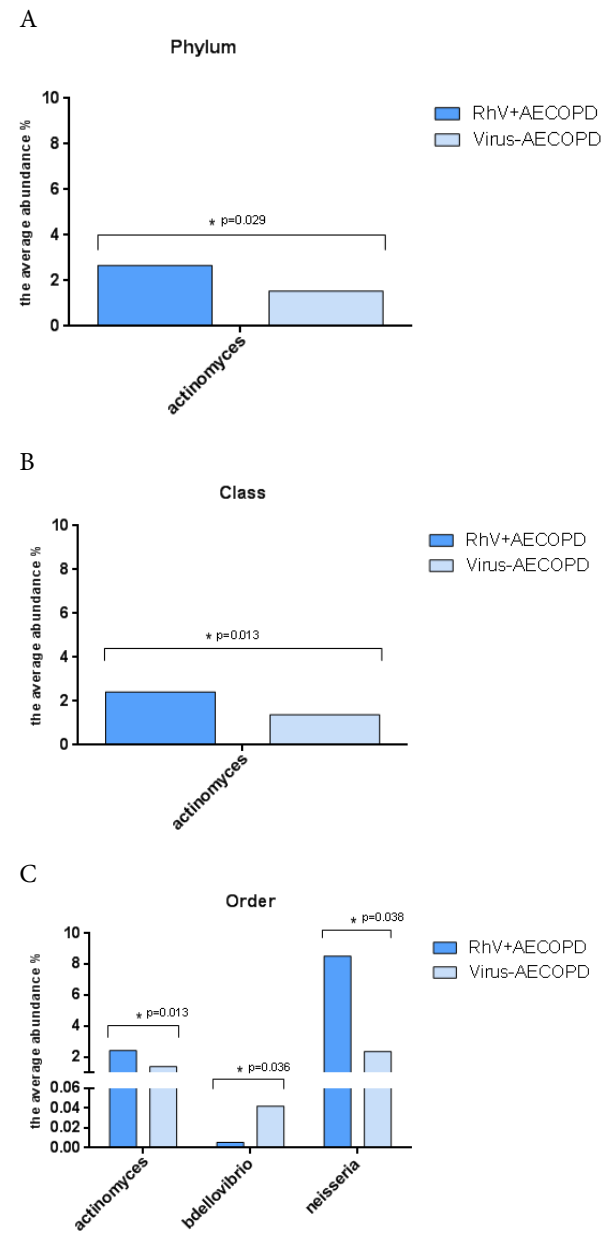
D

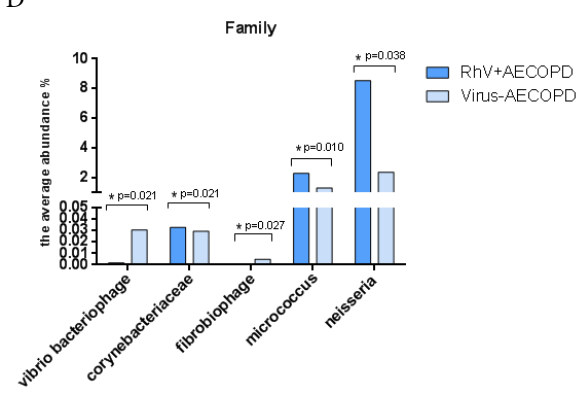

E

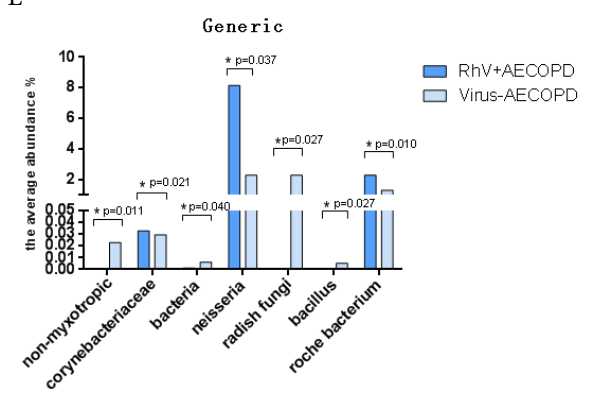

F

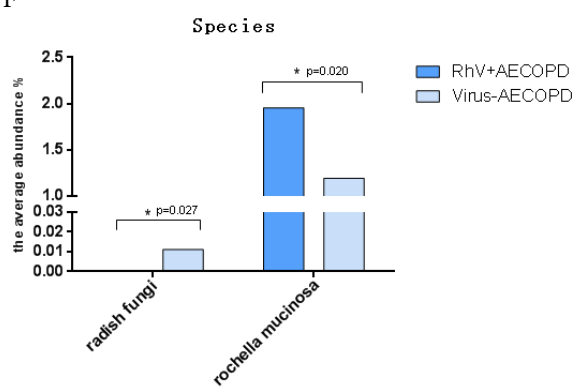

Figure 4. Average bacterial abundance with significant differences between RhV+AECOPD and Virus-AECOPD patients

Note: A-F shows the average abundance of bacteria at different taxonomic levels (A: gate level; B class level; C: order level; D: family level; E: genus level; F: species level); $\mathrm{P}<0.05$ indicates statistical significance.

Abbreviation: AECOPD, acute exacerbation of chronic obstructive pulmonary disease; $\mathrm{RhV}$, rhinovirus.

is mainly based on traditional microbial culture technology, but many bacteria cannot be cultured, it limits identifying bacteria. Compared with the traditional culture methods, the molecular biology methods represented by macro genomics have obvious advantages in detecting bacteria that are difficult to cultivate. ${ }^{9}$

This study explored the effect of rhinovirus on the microbial composition of the lower respiratory tract in COPD patients from a transverse perspective. It was found that there were significant changes in the abundance of many pathogenic and non-pathogenic bacteria after rhinovirus infection. The pathogenic bacteria were Rochella mucosa, belonging to the actinomycetes. Huang et al ${ }^{10}$ also found that there was a significant increase in actinomycetes in sputum specimens of AECOPD patients compared with stabilizers. Molyneaux et al reported ${ }^{3}$ COPD patients were infected with rhinovirus. The results showed that the number of Proteus increased significantly 15 days after rhinovirus infection, and there was significant growth of Haemophilus. This suggests that rhinoviruses may cause significant increases in different pathogenic bacteria in the respiratory tract, but whether they are specific to rhinovirus infection is unknown. Due to the impairment of respiratory immune function in COPD patients, including the removal of damaged mucosal cilia, ${ }^{11}$ increased epithelial infiltration, ${ }^{12}$ dysfunction, ${ }^{13}$ and weakened interferon production, ${ }^{14}$ these deficiencies may lead to inefficient bacterial clearance and allow excessive growth of pathogenic bacteria in the community. It also shows that COPD patients are more likely to cause secondary bacterial infection after virus infection than normal people, which increases the difficulty of acute exacerbation treatment of COPD. It was found that the pathophysiology and mechanism of the local immune response in the respiratory tract are virus-specific. ${ }^{15} \mathrm{~A}$ virus-specific bacterial classification may occur in the respiratory tract. The discovery of specific bacterial infections that depend on any kind of virus will be beneficial to the prevention and treatment of secondary bacterial infections of the respiratory tract caused by the virus in the future. However, up to now, no specific bacterial changes corresponding to different viruses have been clearly identified, which still requires us to continue to study and summarize. ${ }^{16}$

Rochella mucosa found in this study is Gram-positive coccus, belonging to Rochella and Actinomycetes. It has the characteristics of coagulase-negative, sporeless, encapsulated in capsule and facultatively anaerobic. It is usually found in the oral cavity and upper respiratory tract, ${ }^{17-19}$ and occasionally in the gastrointestinal tract, ${ }^{20}$ intestinal epithelium, ${ }^{21}$ breast milk, ${ }^{22}$ and dental plaque..$^{23,24}$ Although Rochella mucosa is considered to be a normal microorganism in the oral and upper respiratory tracts, it is associated with a wide range of diseases, such as bacteremia, central nervous system infection, meningitis, peritonitis, osteomyelitis, necrotizing fasciitis of the neck, endophthalmitis, pneumonia and endocarditis. ${ }^{25-30}$ More and more of them are recognized as opportunistic pathogens, most of which affect the host $^{31}$ of immunodeficiency. Clinical studies have found that single beta-lactam antibiotics or vancomycin or combined with other antibiotics can successfully treat infection caused by Rochella mucosa. ${ }^{32}$ In this study, the local lung defense barrier of COPD patients is damaged, which may provide favorable conditions for the colonization of Rochella mucosa. ${ }^{31}$ Rhinovirus infection aggravates the damage of the barrier and promotes the change of local environment, which may be more conducive to the colonization of the bacteria and eventually lead to secondary bacteria following lower respiratory tract virus infected. ${ }^{33-34}$

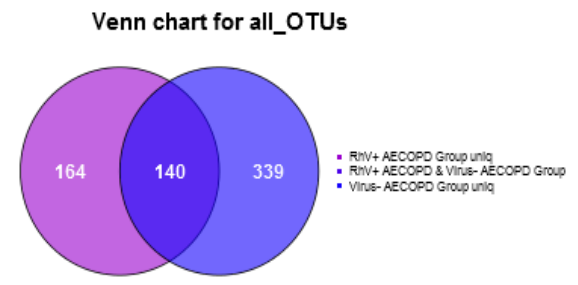

Figure 5. Venn diagram of common/unique OTUs in two groups Note: The interior of each circle symbolically represents the number of OTUs observed in a group; the overlap area will represent the set of OTUs that occur together in the corresponding group; similarly, the single-layer region represents the unique number of OTUs found in a group.

Abbreviation: OTU, Operational Classification Unit.

In this study, the variable V4 region of prokaryotic 16S ribosomal DNA (16S rDNA) gene was sequenced on Illumina MiSeq platform by double exponential two-terminal sequencing. 
The advantage of this sequencing is that its fusion primers are designed, including suitable P5 and P7 Illumina assembly sequences, an $8 \mathrm{nt}$ index sequence and gene-specific primers, which can reduce the cost of long-term customization of primers. In addition, this method can produce a large number of high-quality sequences, which can describe the characteristics of bacterial community composition in the respiratory tract more broadly, and discover the existence of potentially difficult-to-cultivate low-abundance microorganisms. The disadvantage is that the measured microbial data may only reflect the DNA fragments of bacteria rather than live bacteria, which may lead to the data containing partially dead bacteria, and then affect the results of sequencing. ${ }^{13}$

One limitation of this study is that intensive microbiological analysis is performed only in a small number of COPD patient samples from 21 sources. Sequencing results do not fully reflect the changes in the composition of common respiratory microflora caused by rhinovirus infection in COPD population. Another limitation was that the upper and lower respiratory tracts were not tested to see if the microbial changes caused by rhinovirus infection were consistent. Our strengths include controlled rhinovirus research, horizontal research and the use of high-throughput sequencing techniques.

In conclusion, this study investigated the composition of bacterial microorganisms in the lower respiratory tract of patients with rhinovirus-positive AECOPD and virus-negative AECOPD from a transverse point of view by double-index double-end sequencing of the variable V4 region of $16 \mathrm{~S}$ rDNA gene. It was found that there was no significant difference in microbial diversity between the two groups, but there was a significant difference in community structure. We observed a significant difference in actinomycetes between the two groups, and a significant increase in the abundance of Rochella mucosa. Through this sequencing method, we can find the bacterial microorganisms with higher specificity in patients with COPD infected by rhinovirus, and provide guidance for clinical prevention and treatment of secondary bacterial infections.

\section{Conflict of interest statement}

The authors declare they have no conflicts of interest relevant to this manuscript.

\section{Acknowledgments}

We thank Jiawei Gu of Shanghai Medical School, Fudan University, for providing professional English-language editing of this article.

\section{Ethical approval}

Written informed consent was obtained from the patient for publication of this paper. A copy of the written consent is available for review by the Editor-in-Chief of this journal.

\section{REFERENCES}

1 Mathers C, Loncar D. Projections of global mortality and burden of disease from 2002 to 2030. PLoS Med 2006; 3(11): 2011-2030.

2 Pere Almagro, CarmeHernandez, Pable Martinez-Cambor, etal. Seasonality, ambient temperatures and hospitalizations for acute exacerbation of COPD: apopulation-based study in a metropolitan area. Dovepress 2015; 10(1): 899-908.

3 Molyneaux PL, Mallia P, Cox MJ, et al. Outgrowth of the Bacterial Airway Microbiome after Rhinovirus Exacerbation of Chronic Obstructive Pulmonary Disease. Am J Respir Crit Care Med 2013; 188(10): 1224-1231.

4 Hudy MH, Traves SL, Proud D. Transcriptional and Epigenetic Modulation of Human Rhinovirus-Induced CXCL10 Production by Cigarette Smoke. Am J Respir Cell Mol Biol 2014; 50(3): 571-582.

5 Dimopoulos G, Tsiodras S, LerikouM, et al. Viral Profile of COPD Exacerbations According to Patients. The Open Respiratory Medicine Journal 2015; 9(1): 1-8.

6 Blaser MJ, Falkow S. What are the consequences of the disappearing human microbiota? Nat Rev Microbiol 2009; 7(12): 887-894.

7 Mallia P, Footitt J, Sotero R, et al. Rhinovirusinfection induces degradation of antimicrobial peptides and secondarybacterial infection in COPD. Am J Respir Crit Care Med 2012; 186: 1117-1124.

8 Chronic obstructive pulmonary disease study group of Chinese Medical Association.Guidelines for the diagnosis and treatment of chronic obstructive pulmonary disease (2007revision). Chinese Journal of Internal Medicine 2007; 46(3): 254-261.

9 Dickson RP, Erb-Downward JR, Huffnagle GB. The role of the bacterialmicrobiome in lungdisease. Expert Rev Respir Med 2013; 7(3): 245-257.

10 Huang YJ, Sethi S, Murphy T, et al. Airway Microbiome Dynamicsin Exacerbations of Chronic Obstructive Pulmonary Disease. J Clin Microbiol 2014; 52(8): 2813-2823.

11 Verra F, Escudier E, Lebargy F, et al. Ciliaryabnormalities in bronchial epithelium of smokers, ex-smokers, andnonsmokers. Am J Respir Crit Care Med 1995; 151(3): 630-634.

12 Olivera DS, Boggs SE, Beenhouwer C, et al. Cellularmechanisms of mainstream cigarette smoke-induced lung epithelialtight junction permeability changes in vitro. Inhal Toxicol 2007; 19(1): 13-22

13 Donnelly LE, Barnes PJ. Defective phagocytosis in airways disease. Chest 2012; 141(4): 1055-1062.

14 Beasley V, Joshi PV, Singanayagam A, et al. Lung microbiology andexacerbations in COPD. Int J Chron Obstruct Pulmon Dis 2012; 7(1): 555-569.

15 Eccles R. Understanding the symptoms of the common cold andinfluenza. Lancet Infect Dis 2005; 5(11): 718-725.

16 Leopoldo N. Segal, William N. Rom, and Michael D. Weiden.Lung Microbiome for CliniciansNew Discoveries about Bugs in Healthy and Diseased Lungs. Ann Am Thorac Soc Vol 2014; 11(1): 108-116.

17 Bergan T, Bøvre K, Hovig B, et al. Priority of Micrococcus mucilaginosus Migula 1900 over Staphylococcus salivarius: Andrewes and Gordon 1907 with proposal of a neotype strain. International Journal of Systematic Bacteriology 1970; 20(1): 107-113.

18 Olsen I, Preza D, Aas JA, et al. Cultivated and not-yetcultivatedbacteria in oral biofilms. Microbial Ecology in Health and Disease 2009; 21(2): 65-71.

19 Guglielmetti S, Taverniti V, Minuzzo M, et al. Oral bacteria as potential probiotics for the pharyngeal mucosa. Applied and Environmental Microbiology 2010; 76(12): 3948-3958.

20 Wang M, Ahrne S, Jeppsson B, et al. Comparison of bacterial diversity along the human intestinal tract by direct cloning and sequencing of 16S rRNA genes. FEMS Microbiology Ecology 2005; 54(2): 219-231.

21 Jiménez E, Delgado S, Ferna 'ndez L, et al. Assessment of the bacterial diversity of human colostrum and screening of staphylococcal and enterococcal populations for potential virulence factors J . Research in Microbiology, 2008, 159(9): 595-601.

22 Delgado S, Arroyo R, Martı 'n R, et al. PCR-DGGE assessmentof the bacterial diversity of breast milk in women with lactational 
infectiousmastitis. BMC Infectious Diseases 2008; 8(1): 51.

23 Bowden GH. The components of the cell walls and extracellular slime of four strains of Staphylococcus salivarius isolated from human dental plaque. Archives of Oral Biology 1969; 14(6): 685-697.

24 Ready D, Lancaster H, Qureshi F, et al. Effect ofamoxicillin use on oral microbiota in young children. Antimicrobial Agents andChemotherapy 2004; 48(8): 2883-2887.

25 Ramos JM, Mateo I, Rosillo EM, et al. Infection due to Rothia mucilaginosa. A respiratory pathogen? Enferm Infecc Microbiol Clin 2014; 32(5): 206-309.

26 Baeza Martinez C, Zamora Molina L, Garcia Sevila R, et al. Rothia mucilaginosa Pneumonia in an immunocompetent patient. Arch Bronconeumol 2014; 50(11): 493-495.

27 Faiad G, Singh M, Narasimhan A, et al. Rothia mucilaginosa life threatening infections in non-neutropenic hosts. Open J Intern Med 2011; 1(3): 68-71.

28 Rubin SJ, Lyons RW, Murcia AJ, et al. Endocarditis associated with cardiac catheterization due to a Gram-positive coccus designated Micrococcus mucilaginosus incertae sedis. J Clin Microbiol 1978; 7(6): 546-549.
Ascher DP, Zbick C, White C, et al. Infections due to Stomatococcus mucilaginosus: 10 cases and review. Rev Infect Dis 1991; 13(6): 1048-1052.

30 Trevino M, Garcia-Zabarte A, Quintas A, et al. Stomatococcus mucilaginosus septicemia in a patient with acute lymphoblastic leukaemia. Eur J Clin Microbiol Infect Dis 1998; 17(7): 505-507.

31 Maraki S, Papadakis IS. Rothia mucilaginosa pneumonia: a literature review. Infectious Diseases 2015; 47(3): 125-129.

32 Cho EJ, Sung HS, Park SJ, et al. Rothia mucilaginosa Pneumonia Diagnosed by Quantitative Cultures and Intracellular Organisms of Bronchoalveplar Lavage in a Lymphoma Patient. Annals of Laboratory Medicine 2013; 33(2): 145-149.

33 Alotaibi NM, Chen V, Hollander Z, Leipsic JA, Hague CJ, Murphy DT, DeMarco ML, FitzGerald JM, McManus BM, Ng RT, Sin DD. Phenotyping and outcomes of hospitalized COPD patients using rapid molecular diagnostics on sputum samples. Int J Chron Obstruct Pulmon Dis 2019; 14: 311-319.

34 Choi J, Oh JY, Lee YS, Hur GY, Lee SY, Shim JJ, Kang KH, Min KH. Bacterial and Viral Identification Rate in Acute Exacerbation of Chronic Obstructive Pulmonary Disease in Korea. Yonsei Med J 2019; 60(2): 216-222. 